\title{
Bandwidth Partitioning in Decentralized Wireless Networks
}

\author{
Nihar Jindal, Member, IEEE, Jeffrey G. Andrews, Senior Member, IEEE, \\ Steven Weber, Member, IEEE
}

\begin{abstract}
This paper addresses the following question, which is of interest in the design of a multiuser decentralized network. Given a total system bandwidth of $W \mathrm{~Hz}$ and a fixed data rate constraint of $R$ bps for each transmission, how many frequency slots $N$ of size $W / N$ should the band be partitioned into in order to maximize the number of simultaneous links in the network? Dividing the available spectrum results in two competing effects. On the positive side, a larger $N$ allows for more parallel, non-interfering communications to take place in the same area. On the negative side, a larger $N$ increases the SINR requirement for each link because the same information rate must be achieved over less bandwidth. Exploring this tradeoff and determining the optimum value of $N$ in terms of the system parameters is the focus of the paper. Using stochastic geometry, the optimal SINR threshold - which directly corresponds to the optimal spectral efficiency - is derived for both the low SNR (power-limited) and high SNR (interference-limited) regimes. This leads to the optimum choice of the number of frequency bands $N$ in terms of the path loss exponent, power and noise spectral density, desired rate, and total bandwidth.
\end{abstract}

\section{INTRODUCTION}

For purposes of wireless communication, the electromagnetic spectrum is typically first divided into a large number of bands by regulatory agencies such as the FCC or the European Commission. These bands are typically allocated by executive fiat or auction, and for particular purposes. Once allocated, these bands are usually further divided into many smaller bands that individual users have access to. This entire process has a major impact on the efficiency with which spectral resources are used, but historically appears to have been done in a mostly ad hoc manner. This paper attempts to develop a theoretical basis for bandwidth partitioning, in particular the second partitioning of an allocated band into subbands.

To be more specific, consider a spatially distributed wireless network, representing either an ad hoc network or an unlicensed (and uncoordinated) spectrum system, e.g., 802.11. In such systems it is common to have a fixed total bandwidth, a large number of potential users, and a limit on acceptable packet loss rates. It is also typical to have a target data rate for each user, either to support a certain application or due to user expectations. This gives rise to the following basic question: given bandwidth $W$ and a fixed rate requirement $R$ for each transmitter-receiver link in the network, how many slots $N$ of size $W / N$ should this band be partitioned into in order to maximize the number of links (i.e., spatial density of transmissions) that can achieve this rate $R$ at a specified outage probability (i.e., packet error rate)?

For example, given $1 \mathrm{MHz}$ of bandwidth and a desired rate of $1 \mathrm{Mbps}$, should (a) each transmitter utilize the entire spectrum and thus require an SINR of 1 (utilizing $R=W \log _{2}(1+\mathrm{SINR}$ ) bits/sec), (b) the band be split into two $0.5 \mathrm{MHz}$ sub-bands where each transmitter utilizes one of the sub-bands with a required SINR of 3, or (c) the band be split into $N>2$ orthogonal $\frac{1}{N} \mathrm{MHz}$ sub-bands where each transmitter utilizes one of the sub-bands with a required SINR of $2^{N}-1$ ?

Increasing the number of sub-bands $N$ has two competing effects. On the positive side, it allows for parallel, non-interfering communications on different sub-bands. On the negative side, transmitting at the same data rate over less bandwidth requires each transmission to be performed at a higher spectral efficiency ( $R$ bps over $\frac{W}{N}$ $\mathrm{Hz}$ corresponds to a spectral efficiency of $\frac{R}{W / N} \mathrm{bps} / \mathrm{Hz}$ ), which translates to a higher SINR requirement and thus

The authors are with the ECE Departments of the University of Minnesota, the University of Texas at Austin, and Drexel University, respectively. e-mail: nihar@umn.edu, jandrews@ece.utexas.edu, sweber@ece.drexel.edu.

This work is supported under an NSF collaborative research grant awarded to the three authors (NSF grant \#0635003 (Weber), \#0634979 (Andrews), and \#0634763 (Jindal)), and by the DARPA IT-MANET program, Grant W911NF-07-1-0028 (Andrews, Jindal, Weber). Earlier versions of this work appeared at the ITA workshop [1] and ISIT [2] in Jan. and June 2007, respectively. 
a larger interference-free area. The objective of this paper is understanding this tradeoff and characterizing the optimum value of $N$ in terms of the system parameters.

\section{A. Technical Approach}

To allow for analytical tractability, we optimize the number of sub-bands for a network consisting of transmitterreceiver pairs distributed on the two-dimensional plane. More specifically, the network we consider has the following key characteristics:

- Transmitter locations are a realization of a homogeneous spatial Poisson process.

- Each transmitter communicates with a single receiver that is a distance $d$ meters away.

- All transmissions occur at power $\rho$ and rate $R$ bits/sec, the noise spectral density is $N_{0}$, and attenuation follows path-loss exponent $\alpha$.

- The system bandwidth of $W \mathrm{~Hz}$ is divided into $N$ equal sub-bands of $\frac{W}{N} \mathrm{~Hz}$, and each transmission occurs on a randomly chosen sub-band.

- Each receiver treats multi-user interference as noise, and thus a transmission is successful if and only if the received SINR is larger than a threshold determined by $R, W$, and $N$.

The second to last assumption should make it clear that we are considering only an off-line optimization of the frequency band structure, and that no on-line (e.g., channel- and queue-based) transmission or sub-band decisions are considered.

By considering such a network, tools from stochastic geometry can be used to characterize the distribution of received interference and thus to quantify the success probability of each transmission as a function of the transmitter density and the SINR threshold. In this context, the question at hand is determining the value of $N$ that maximizes success probability for a given spatial density of transmitters. Rather than considering the optimization in terms of $N$, it is convenient to pose the problem in terms of the spectral efficiency of each communication $\frac{R}{W / N}$. Our main result is an exact characterization of the optimal spectral efficiency in the form of a simple fixed point equation.1 Furthermore, the optimal spectral efficiency is seen to be a function only of the path-loss exponent and the energy per information bit $\frac{E_{b}}{N_{0}}=\frac{P}{N_{0} R}$ (where $P$ is the received power, $N_{0}$ is the noise spectral density, and $R$ is the rate [3]), and thus is independent of the transmitter density. In order for a network to operate optimally, $N$ should be increased until the spectral efficiency $\left(\frac{N R}{W}\right)$ is equal to its optimal value.

When thermal noise is negligible relative to the received signal power (i.e., $\frac{E_{b}}{N_{0}} \rightarrow \infty$ ), the network is purely interference-limited and the optimal spectral efficiency is a function of the path loss exponent $(\alpha)$ alone. For reasonable path loss exponents the optimal spectral efficiency lies between the low-SNR and high-SNR regimes. For example, the optimal is $1.3 \mathrm{bps} / \mathrm{Hz}$ (SINR threshold of $1.6 \mathrm{~dB}$ ) and $2.3 \mathrm{bps} / \mathrm{Hz}$ (SINR threshold of $5.9 \mathrm{~dB}$ ) for $\alpha=3$ and $\alpha=4$, respectively. When thermal noise is not negligible (i.e., $\frac{E_{b}}{N_{0}}$ is small), the optimal spectral efficiency is shown to be the fraction $\left(1-\frac{2}{\alpha}\right)$ of the maximum spectral efficiency achievable in the absence of interference.

Increasing $N$, which corresponds to decreasing the bandwidth and increasing the area consumed by each transmission, is seen to be beneficial as long as area (i.e., the SINR threshold) increases at a reasonable rate with $N$. For interference-limited networks this is true until the high-SNR regime is reached, at which point a huge SINR increase is required for any additional bandwidth reduction. For power-limited networks this is true until the SINR threshold approaches the interference-free SNR, at which point the system becomes overly sensitive to interference.

\section{B. Related Work}

The problem studied in this work is essentially the optimization of frequency reuse in uncoordinated decentralized networks, which is a well studied problem in the context of centrally-planned cellular and other hierarchical networks; see for example [4], [5], [6] and references therein. In both settings the tradeoff is between the bandwidth utilized per cell or transmission - which is inversely proportional to the frequency reuse factor - and the achieved $\mathrm{SINR} /$ spectral efficiency per transmission. A key difference is that regular frequency reuse patterns can be used in cellular networks, whereas in an ad hoc or unlicensed network this is impossible. Another crucial difference

\footnotetext{
${ }^{1}$ Because SINR is a function of spectral efficiency, this is equivalent to a derivation of the optimal SINR threshold.
} 
is in terms of analytical tractability. Although there has been a tremendous amount of work on frequency reuse optimization for cellular networks, these efforts generally do not lead to clean analytical results. On the contrary, in this work we are able to derive simple analytical results for decentralized networks that cleanly show the dependence of the optimal reuse factor on basic system parameters.

A number of works have considered related problems in the context of decentralized networks, although none appear to have investigated the optimization considered here. In [7] the time-bandwidth-area product achieved by different codes are evaluated. This metric is essentially equivalent to the inverse of transmission density in our network model, but the authors do not pursue optimization of this metric, which is the essence of our work. In [8], the authors jointly optimize rate, transmitter-receiver distance $(d)$, and density in order to maximize the transport capacity (i.e., product of rate and distance) of a random-access network. This setting is very different from our framework in which we assume a fixed rate and transmitter-receive distance, and as a result conclusions differ significantly. For example, the optimum SINR threshold in [8] for some networks is found to be orders of magnitude smaller than $0 \mathrm{~dB}$, whereas we find optimal values around $0 \mathrm{~dB}$. In [9] a network consisting of a large number of interfering transmitter-receiver pairs is analyzed, but no spatial model is used and only fading is considered. In [10] the issue of frequency reuse is considered in a one-dimensional, evenly spaced, multi-hop wireless network. Some similar general insights are derived, but the regular spacing of interferers seems to prevent derivation of clean analytical results as is possible for the 2-D network considered here. In a recent contribution the interactions between multiple random-access networks have been considered from a game-theoretic perspective [11], and portions of the analysis of a single network in [11] coincide with our initial findings reported earlier in [1]. There has also been a good deal of work on multi-channel wireless networks, but this body of work generally deals with scheduled networks as opposed to our treatment of unscheduled networks (see [12] and references therein). Perhaps most relevant is [13], in which algorithms for dynamic allocation of bandwidth-area resources are proposed.

\section{Preliminaries}

\section{A. Network Model}

We consider a set of transmitting nodes at an arbitrary snapshot in time with locations specified by a homogeneous Poisson point process (PPP) of intensity $\lambda$ on the infinite 2-D plane. All nodes are assumed to simultaneously transmit with power $\rho$. By the stationarity of the PPP it is sufficient to analyze the behavior of a single reference TX-RX pair (TX 0, RX 0), separated by assumption by a distance $d$. Note that the receivers are not a part of the transmitter process. From the perspective of RX 0, the interferers follow the distribution of the PPP conditioned on the location of TX 0 (referred to as the Palm distribution). However, by Slivnyak's theorem [14] this distribution is the same as the unconditional distribution and therefore the locations of the interfering nodes form a homogeneous PPP of intensity $\lambda$. Received power is modeled by path loss with exponent $\alpha>2$. If $X_{i}$ denotes the distance of the $i$-th transmitting node to the reference receiver and the transmit signal of the $i$-th transmitter is denoted as $U_{i}$, the reference received signal is:

$$
Y_{0}=U_{0} d^{-\alpha / 2}+\sum_{i \in \Pi(\lambda)} U_{i} X_{i}^{-\alpha / 2}+Z_{i}
$$

where $Z_{i}$ is additive Gaussian noise with power $\eta$. The resulting SINR therefore is:

$$
\operatorname{SINR}_{0}=\frac{\rho d^{-\alpha}}{\eta+\sum_{i \in \Pi(\lambda)} \rho X_{i}^{-\alpha}},
$$

where $\Pi(\lambda)$ indicates the point process describing the (random) interferer locations. If Gaussian signaling is used, the received mutual information (conditioned on interferer locations) is $I\left(U_{0} ; Y_{0} \mid \Pi(\lambda)\right)=\log _{2}\left(1+\mathrm{SINR}_{0}\right)$ bits/symbol. In the fixed rate setting considered here, the probability the received mutual information is smaller than the transmission rate is known to be a good approximation to packet error rate if strong channel coding is used [15], and thus is the primary metric in this work.

A few comments in justification of our model are in order. Although the model contains many simplifications to allow for tractability, it contains many of the critical elements of a real decentralized network. First, the spatial Poisson distribution means that transmitting nodes are randomly and independently located; this is reasonable in a network with indiscriminate node placement or substantial mobility assuming that no intelligent transmission 
scheduling is performed Scheduling generally attempts to ensure that simultaneous transmissions are sufficiently separated in space, and thus can significantly change the spatial distribution of simultaneous transmissions. However, even simple scheduling protocols can incur considerable overhead and latency and thus unscheduled systems (or systems using ALOHA-like protocols that make transmission decisions independent of interference conditions) as considered here are of interest. This is particularly true when scheduling overhead begins to overtake the advantage of scheduling, as may be the case with high mobility or very bursty traffic. The assumptions of fixed TX-RX distances and no fading are often not reasonable, but as we discuss in Section VI-B our results also apply to networks with fading and/or variable distances in the interference-limited regime (no thermal noise). Furthermore, our results are reasonably accurate in the presence of non-negligible thermal noise when the fading and distance variation is not too large. Finally, we note that fixed- rather than variable-rate communication is appropriate for some, but not necessarily all, settings, e.g., single-hop communication with very stringent delay constraints. In other settings (e.g., when delay constraints are less stringent) variable rate communication is more appropriate; optimizing bandwidth partitioning in this context is of interest but is outside the scope of this work.

\section{B. Outage Probability/Maximum Density Characterization}

An outage occurs whenever the SINR falls below threshold $\beta$, or equivalently whenever the received mutual information is smaller than $\log _{2}(1+\beta)$. Therefore, the system-wide outage probability is:

$$
P_{\text {out }}(\lambda, \beta, \eta) \triangleq \mathbb{P}\left(\frac{\rho d^{-\alpha}}{\eta+\sum_{i \in \Pi(\lambda)} \rho X_{i}^{-\alpha}} \leq \beta\right) .
$$

This quantity is computed over the distribution of transmitter positions and is an increasing function of the intensity $\lambda$. The SINR threshold $\beta$ and the noise power $\eta$ are treated as constants here, but are related to $R, W$, and $N$ in the following section. Random variable $X$ is defined as the received interference raised to the power $-\frac{2}{\alpha}$ :

$$
X \triangleq\left(\sum_{i \in \Pi(\lambda)} X_{i}^{-\alpha}\right)^{-\frac{2}{\alpha}}
$$

which allows the outage probability to be written in terms of $X$ as:

$$
P_{\text {out }}(\lambda, \beta, \eta)=\mathbb{P}\left(\frac{\rho d^{-\alpha}}{\eta+\rho X^{-\frac{\alpha}{2}}} \leq \beta\right)=\mathbb{P}\left(X \leq d^{2}\left(\frac{1}{\beta}-\frac{\eta}{\rho d^{-\alpha}}\right)^{-\frac{2}{\alpha}}\right) .
$$

It is useful to write this expression in terms of a normalized interferer process. If we define $Z$ as the received interference for a process with intensity $\frac{1}{\pi}$ :

$$
Z \triangleq\left(\sum_{i \in \Pi(1 / \pi)} Z_{i}^{-\alpha}\right)^{-\frac{2}{\alpha}}
$$

and note that a PPP with intensity $\lambda$ is equivalent to a PPP with intensity $\frac{1}{\pi}$ scaled by $\frac{1}{\sqrt{\pi \lambda}}$, it follows that $X$ and $\frac{1}{\pi \lambda} Z$ have the same distribution. Therefore

$$
P_{\text {out }}(\lambda, \beta, \eta)=F_{Z}\left(\lambda \pi d^{2}\left(\frac{1}{\beta}-\frac{\eta}{\rho d^{-\alpha}}\right)^{-\frac{2}{\alpha}}\right)
$$

where $F_{Z}(\cdot)$ denotes the $\mathrm{CDF}$ of random variable $Z$. Although a closed form expression for $F_{Z}(\cdot)$ is not known except for the special case of $\alpha=4$ [16], this characterization of the outage probability allows us to derive an exact solution to the bandwidth partition problem.

In many scenarios, the network is subject to an outage constraint and the quantity of interest is the maximum intensity of attempted transmissions $\lambda_{\epsilon}$ such that the outage probability (for a fixed $\beta$ ) is no larger than $\epsilon$. Because outage probability increases monotonically with density, an expression for $\lambda_{\epsilon}$ is reached by inverting (1):

$$
\lambda_{\epsilon}=\frac{F_{Z}^{-1}(\epsilon)}{\pi d^{2}}\left(\frac{1}{\beta}-\frac{\eta}{\rho d^{-\alpha}}\right)^{\frac{2}{\alpha}}
$$




\begin{tabular}{|l|l|}
\hline Parameter & Description \\
\hline$R$ & Transmission Rate (bits/sec) \\
\hline$W$ & Total System Bandwidth (Hz) \\
\hline$\rho$ & Transmission Power \\
\hline$N_{0}$ & Noise Spectral Density \\
\hline$d$ & Transmitter-Receiver Distance \\
\hline$\frac{E_{b}}{N_{0}}=\frac{\rho d^{-\alpha}}{N_{0} R}$ & Energy per Information Bit \\
\hline$\epsilon$ & Outage Constraint \\
\hline$N$ & Number of Sub-Bands \\
\hline$\beta$ & SINR Threshold \\
\hline
\end{tabular}

TABLE I

SUMMARY OF SYSTEM PARAMETERS

where $F_{Z}^{-1}(\cdot)$ is the inverse of $F_{Z}(\cdot)$.

Because the SINR is upper bounded by only considering the contribution of the nearest interferer, a necessary (but not sufficient) condition for successful communication is that a circle centered about the receiver of area $\pi d^{2}\left(\frac{1}{\beta}-\frac{\eta}{\rho d^{-\alpha}}\right)^{-\frac{2}{\alpha}}$ be free of interferers [17]. On the other hand, the effective area consumed by each transmission when an outage level of $\epsilon$ is required is the inverse of the density $\lambda_{\epsilon}$ :

$$
\frac{1}{\lambda_{\epsilon}}=\frac{1}{F_{Z}^{-1}(\epsilon)} \pi d^{2}\left(\frac{1}{\beta}-\frac{\eta}{\rho d^{-\alpha}}\right)^{-\frac{2}{\alpha}},
$$

which is the interferer-free area from the necessary condition above multiplied by the constant $\frac{1}{F_{z}^{-1}(\epsilon)}$. This constant factor, which increases without bound as $\epsilon \rightarrow 0$ and which is larger than one for all but the largest values of $\epsilon$, accounts for the fact that transmitters are randomly located and can be intuitively thought of as a back-off parameter that ensures the outage constraint is met. This interpretation turns out to be useful when interpreting bandwidth partitioning in terms of bandwidth and area.

\section{Problem Formulation AND General Solution}

We are now able to address the problem of interest, which is determining the number of sub-bands that maximize the density of transmissions such that the outage probability is no larger than $\epsilon$. As made explicit at the end of this section, finding the value of $N$ that minimizes outage probability for a fixed total density of transmitters is the dual of this problem and has precisely the same solution. For the reader's reference, the relevant system parameters are summarized in Table I

If the system bandwidth is not split $(N=1)$, each node utilizes the entire bandwidth of $W \mathrm{~Hz}$. The SINR required ( $\beta$ ) to achieve a rate of $R$ bps is determined by inverting the AWGN capacity expression $R=W \log _{2}(1+\beta)$, which gives $\beta=2^{\frac{R}{W}}-1$. The maximum intensity of transmissions can be determined by evaluating (2) with this value of $\beta$ and $\eta=N_{0} W$. If the system bandwidth is split into $N>1$ orthogonal sub-bands each of width $\frac{W}{N}$, and each transmitter-receiver pair uses one randomly selected sub-band, the required SINR $\beta(N)$ is determined by inverting the rate expression:

$$
R=\frac{W}{N} \log _{2}(1+\beta(N)) \rightarrow \beta(N)=2^{\frac{N R}{W}}-1 .
$$

Because each transmitter randomly chooses a sub-band, the users on each sub-band are still a PPP and are independent across bands. As a result, the maximum intensity of transmissions per sub-band is $\lambda_{\epsilon}$ as defined in (2) with SINR threshold $\beta(N)$ and noise power $\eta=N_{0} \frac{W}{N}$. Since the $N$ sub-bands are statistically identical, the maximum total intensity of transmissions, denoted $\lambda_{\epsilon}^{T}$, is the per sub-band intensity $\lambda_{\epsilon}$ multiplied by $N$. Therefore, from (2) we have:

$$
\lambda_{\epsilon}^{T}(N)=N\left(\frac{F_{Z}^{-1}(\epsilon)}{\pi d^{2}}\right)\left(\frac{1}{\beta(N)}-\frac{N_{0}\left(\frac{W}{N}\right)}{\rho d^{-\alpha}}\right)^{\frac{2}{\alpha}} .
$$


The optimal number of sub-bands $N^{*}$ is that which maximizes total transmission density:

$$
N^{*}=\arg \max _{N} \lambda_{\epsilon}^{T}(N) .
$$

It is useful to interpret this optimization in terms of bandwidth and area. Dividing (5) by $W$ and then inverting yields:

$$
\frac{W}{\lambda_{\epsilon}^{T}(b)}=\frac{1}{F_{Z}^{-1}(\epsilon)} \underbrace{\left(\frac{W}{N}\right)}_{\text {Bandwidth }} \underbrace{\pi d^{2}\left(\frac{1}{\beta(N)}-\frac{N_{0} W}{N \rho d^{-\alpha}}\right)^{-\frac{2}{\alpha}}}_{\text {Interferer-Free Area }} .
$$

which is the product of the constant $\frac{1}{F_{Z}^{-1}(\epsilon)}$, sub-band bandwidth $\frac{W}{N}$, and the required interferer-free area. Total density is maximized by minimizing this quantity, i.e., by minimizing the bandwidth-area product of each transmission. It is easily checked that the interferer-free area is a strictly increasing function of $N$. Thus, as the number of sub-bands $N$ is increased the bandwidth consumed by each transmission decreases while the area increases, leading to a non-trivial tradeoff.

Rather than solving the maximization in (6) with respect to $N$, it is more convenient to maximize with respect to the operating spectral efficiency, which is equal to the transmission rate divided by the bandwidth of each sub-band:

$$
b \triangleq \frac{R}{W / N} \text { bps/Hz. }
$$

It is important to note that the operating spectral efficiency $b$ is a design parameter even though the per-transmission rate $R$ and system bandwidth $W$ are fixed. 2

With this substitution the transmission density can be written as a function of $b$ :

$$
\lambda_{\epsilon}^{T}(b)=\left(\frac{F_{Z}^{-1}(\epsilon)}{\pi d^{2}}\right)\left(\frac{W}{R}\right) b\left(\frac{1}{2^{b}-1}-\frac{1}{b} \frac{N_{0} R}{\rho d^{-\alpha}}\right)^{\frac{2}{\alpha}}
$$

Noting that the constant $\frac{\rho d^{-\alpha}}{N_{0} R} \triangleq \frac{E_{b}}{N_{0}}$ is the received energy per information bit [3] and defining the constant $\kappa \triangleq\left(\frac{F_{Z}^{-1}(\epsilon)}{\pi d^{2}}\right)\left(\frac{W}{R}\right)$, this can be further simplified as:

$$
\lambda_{\epsilon}^{T}(b)=\kappa b\left(\frac{1}{2^{b}-1}-\frac{1}{b \frac{E_{b}}{N_{0}}}\right)^{\frac{2}{\alpha}} .
$$

The optimal spectral efficiency $b^{*}$ is therefore the solution to the following optimization:

$$
b^{*}=\arg \max _{b>0} b\left(\frac{1}{2^{b}-1}-\frac{1}{b \frac{E_{b}}{N_{0}}}\right)^{\frac{2}{\alpha}} .
$$

Note that the optimal $b^{*}$ depends only on the path loss exponent $\alpha$ and $\frac{E_{b}}{N_{0}}$, and thus any dependence on power and rate is completely captured by $\frac{E_{b}}{N_{0}}$. By posing the problem in terms of spectral efficiency, any direct dependence on $W$ is removed. Furthermore, the problem is completely independent of the outage constraint $\epsilon$.

The problem in (11) is only feasible for $b$ satisfying $\frac{1}{2^{b}-1}-\frac{1}{b \frac{E_{b}}{N_{0}}} \geq 0$, which corresponds to the SINR threshold $\beta=2^{b}-1$ being no larger than the interference-free $\operatorname{SNR} \frac{N \rho d^{-\alpha}}{N_{0} W}$. Some simple manipulation shows that this condition is equivalent to $b \leq \mathrm{C}\left(\frac{E_{b}}{N_{0}}\right)$, where $\mathrm{C}\left(\frac{E_{b}}{N_{0}}\right)$ is the maximum spectral efficiency of an AWGN channel and thus is the solution to [3, Equation 23]:

$$
{ }_{2}^{\mathrm{C}\left(\frac{E_{b}}{N_{0}}\right)}-1=\frac{E_{b}}{N_{0}} \mathrm{C}\left(\frac{E_{b}}{N_{0}}\right) .
$$

\footnotetext{
${ }^{2}$ If only bandwidth optimization is considered, $b$ should be limited to integer multiples of $\frac{R}{W}$; in this case $N^{*}$ is either the integer floor or ceiling of $b^{*}\left(\frac{R}{W}\right)$ due to the nature of the objective function. However, if a more general scenario is considered where the sub-band structure as well as the length of transmission is being designed (e.g., in a packetized system), these two parameters allow for operation at any desired $b$. Therefore, arbitrary $b>0$ are considered for the remainder of the paper.
} 


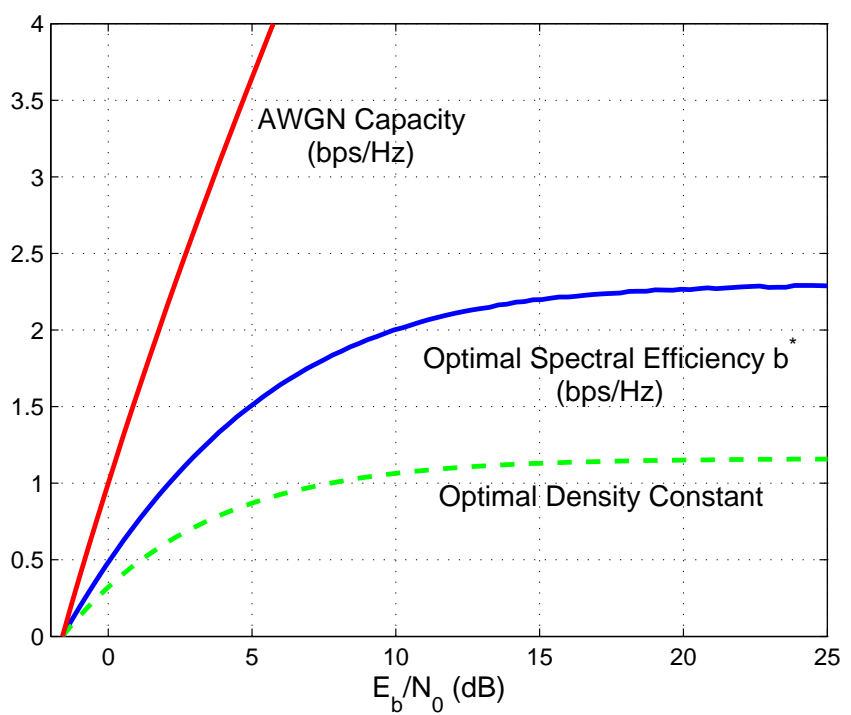

Fig. 1. Optimal Spectral Efficiency $b^{*}$ and Optimal Density Constant $\frac{\lambda_{\epsilon}^{T}\left(b^{*}\right)}{\kappa}$ vs. $\frac{E_{b}}{N_{0}}$ for $\alpha=4$.

The domain of the maximization is thus $0 \leq b \leq \mathrm{C}\left(\frac{E_{b}}{N_{0}}\right)$. If $\frac{E_{b}}{N_{0}} \leq \log _{e} 2=-1.59 \mathrm{~dB}$ the problem is infeasible for any $b$ because this corresponds to operating beyond interference-free capacity 3 .

By taking the derivative of $\lambda_{\epsilon}^{T}(b)$ and setting it equal to zero, the optimal spectral efficiency $b^{*}$ can be characterized in terms of a fixed point equation parameterized by $\alpha$ and $\frac{E_{b}}{N_{0}}$ :

Theorem 1: The optimum operating spectral efficiency $b^{*}$ is the unique positive solution of the following equation:

$$
\frac{E_{b}}{N_{0}} b\left(2^{b}-1\right)-\frac{E_{b}}{N_{0}} \frac{2}{\alpha} b^{2} 2^{b} \log _{e} 2-\left(1-\frac{2}{\alpha}\right)\left(2^{b}-1\right)^{2}=0
$$

Furthermore, $b^{*}$ is an increasing function of $\frac{E_{b}}{N_{0}}$ and of $\alpha$.

Proof: See Appendix 【

Although we are not able to find a general closed-form expression for (13), this expression is easily solved numerically and we can find closed form solutions in the asymptotic regimes $\left(\frac{E_{b}}{N_{0}} \rightarrow \infty\right.$ and $\left.\frac{E_{b}}{N_{0}} \rightarrow-1.59 \mathrm{~dB}\right)$. In Fig. 1 the numerically computed optimum spectral efficiency $b^{*}$ and the corresponding density constant $\frac{\lambda_{\epsilon}^{T}\left(b^{*}\right)}{\kappa}$ are plotted versus $\frac{E_{b}}{N_{0}}$ for $\alpha=4$, along with the spectral efficiency of an interference-free AWGN channel $C^{\kappa}\left(\frac{E_{b}}{N_{0}}\right)$. From this figure, two asymptotic regimes of interest can be identified:

- Interference-Limited Networks: When $\frac{E_{b}}{N_{0}}$ is sufficiently large, the effect of thermal noise vanishes and performance depends only on multi-user interference. As a result, the optimal $b^{*}$ and density $\lambda_{\epsilon}^{T}\left(b^{*}\right)$ both converge to constants as $\frac{E_{b}}{N_{0}} \rightarrow \infty$.

- Power-Limited Networks: When $\frac{E_{b}}{N_{0}}$ is close to its minimum value of $-1.59 \mathrm{~dB}, b^{*}$ and $\lambda_{\epsilon}^{T}\left(b^{*}\right)$ scale linearly with $\frac{E_{b}}{N_{0}}(\mathrm{~dB})$ and show characteristics very similar to AWGN spectral efficiency [3].

In Section IV] the interference-limited regime is explored and a closed form expression for the optimal value of $b^{*}$ in terms of only the path-loss exponent is derived. Once a system is in this regime, performance is virtually unaffected by further increasing transmission power. In Section $\nabla$ the power-limited regime is explored and simple

\footnotetext{
${ }^{3}$ For readers less familiar with the power-limited regime, note that fixing power $P$ and noise spectral density $N_{0}$ and using less bandwidth leads to a decreasing rate, i.e., the function $w \log _{2}\left(1+\frac{P}{N_{0} w}\right) \downarrow 0$ as $w \rightarrow 0$. Thus, there is a minimum bandwidth needed to achieve a particular rate $R$ even in the absence of multi-user interference; this is the solution to $R=w \log _{2}\left(1+\frac{P}{N_{0} w}\right)$ and is precisely the quantity $\frac{R}{\mathrm{C}\left(\frac{E_{b}}{N_{0}}\right)}$. Furthermore, note that $w \log _{2}\left(1+\frac{P}{N_{0} w}\right) \uparrow\left(\frac{P}{N_{0}}\right) \log _{e} 2$ as $w \rightarrow \infty$; therefore the minimum energy per information bit $\frac{E_{b}}{N_{0} \text { min }}=\frac{P}{N_{0} R}=\log _{e} 2=-1.59 \mathrm{~dB}$ and $\mathrm{C}\left(\log _{e} 2\right)=0$.
} 


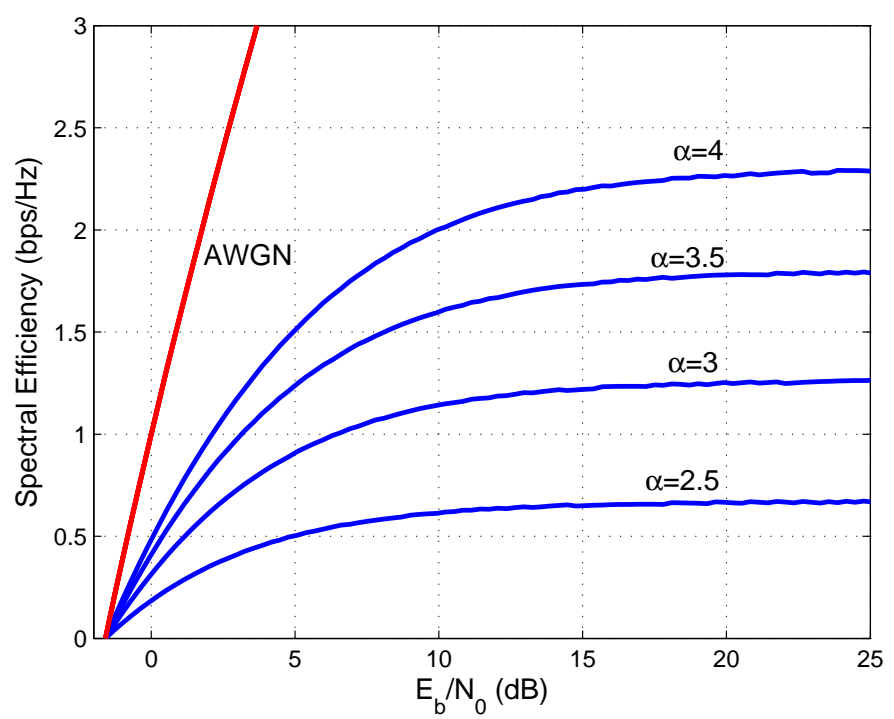

Fig. 2. Optimal Spectral Efficiency $b^{*}$ vs. $\frac{E_{b}}{N_{0}}$ for $\alpha=2.5,3,3.5,4$.

expressions for $b^{*}$ and $\lambda_{\epsilon}^{T}\left(b^{*}\right)$ in terms of $\alpha$ and $\frac{E_{b}}{N_{0}}$ are given that are accurate for $\frac{E_{b}}{N_{0}}$ near $-1.59 \mathrm{~dB}$. Although intuition might suggest that noise is dominant and thus interference is negligible in this regime, this is not the case as evidenced by the fact that the optimum spectral efficiency $b^{*}$ is considerably smaller than the interference-free spectral efficiency $\mathrm{C}\left(\frac{E_{b}}{N_{0}}\right)$. Furthermore, increasing transmission power does significantly increase density in this regime. Between these two regimes (approximately from 2-3 dB to 15-20 dB), $b^{*}$ increases sub-linearly with $\frac{E_{b}}{N_{0}}$ $(\mathrm{dB})$ and the intuition is a combination of the insights derived for the interference- and power-limited regimes.

In Fig. 2 numerically computed values of $b^{*}$ are plotted versus $\frac{E_{b}}{N_{0}}$ for $\alpha=2.5,3,3.5$ and 4 , and the interferencelimited regime is seen to begin around $15 \mathrm{~dB}$ for each value of $\alpha$. Although not visible here, it is interesting to note that $\frac{\lambda_{\epsilon}^{T}\left(b^{*}\right)}{\kappa}$ is not monotonic with respect to $\alpha$; on the other hand, it is easily verified that $\lambda_{\epsilon}^{T}\left(b^{*}\right)$ monotonically increases with $\frac{E_{b}}{N_{0}}$.

Remark 3.1: The dual problem of density maximization subject to an outage constraint is outage minimization for a given density. In this case the overall outage probability is the same as the outage probability on each of the $N$ sub-bands, each of which has density $\frac{\lambda}{N}$. Substituting appropriate values for the SINR threshold and the noise power in (1) yields:

$$
P_{\text {out }}(b)=F_{Z}\left(\lambda \pi d^{2}\left(\frac{R}{W}\right)\left(\frac{1}{b}\right)\left(\frac{1}{2^{b}-1}-\frac{1}{b \frac{E_{b}}{N_{0}}}\right)^{-\frac{2}{\alpha}}\right) .
$$

Outage probability is minimized by minimizing the argument of the CDF (due to the non-decreasing nature of any $\mathrm{CDF}$ ). Because the argument is inversely proportional to the argument of the maximization in (11), the problems of outage minimization and density maximization are equivalent. To understand the impact of partitioning, it is useful to note that $F_{Z}(z)$ is approximately linear for small $z$ [17].

Remark 3.2: If the available transmission rates are at a gap to capacity, i.e., $R=W \log _{2}\left(1+\Gamma^{-1}\right.$. SINR) for some $\Gamma>1$, the required SINR increases by a factor of $\Gamma$ to $\beta(N)=\Gamma\left(2^{N R / W}-1\right)$ and the density is given by $\lambda_{\epsilon}^{T}(b)=\Gamma^{-\frac{2}{\alpha}} \kappa b\left(\frac{1}{2^{b}-1}-\frac{1}{b \frac{E_{b}}{N_{0}}}\right)^{\frac{2}{\alpha}}$ where $\frac{E_{b}}{N_{0}}=\frac{\rho d^{-\alpha}}{\Gamma N_{0} R}$. Thus, the optimal spectral efficiency is given by evaluating Theorem 1 with $\frac{E_{b}}{N_{0}}=\frac{\rho d^{-\alpha}}{\Gamma N_{0} R}$. 


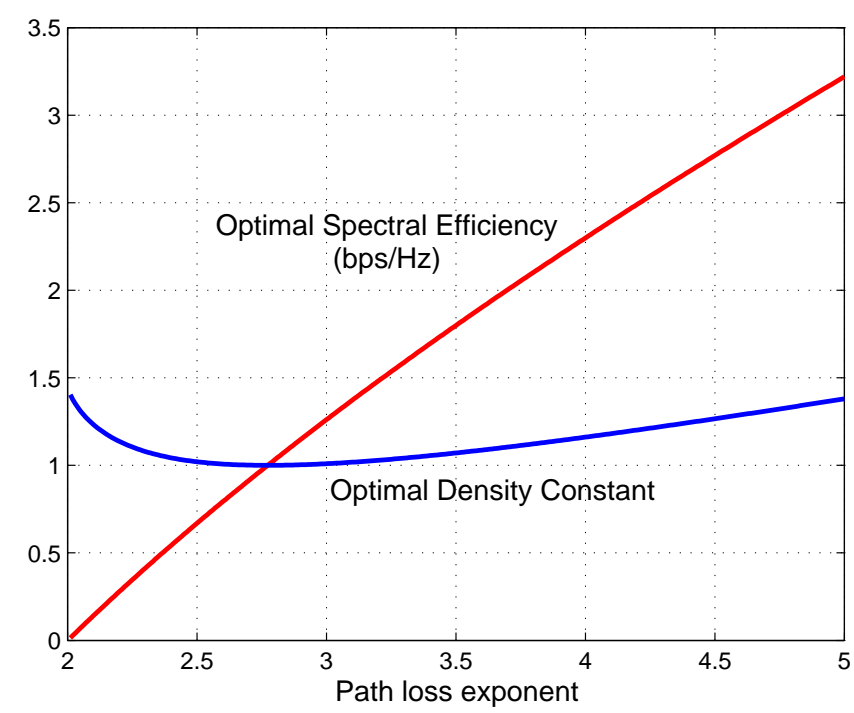

Fig. 3. Optimal Spectral Efficiency $b^{*}$ and Optimal Density Constant $\frac{\lambda_{\epsilon}^{T}\left(b^{*}\right)}{\kappa}$ vs. Path Loss Exponent $\alpha$ for Interference-Limited Networks.

\section{PARTitioning FOR InTERFEREnCE-Limited Networks}

In systems with sufficiently powered devices (i.e., large $\frac{E_{b}}{N_{0}}$ ), thermal noise is essentially negligible. In the limiting case where $N_{0}=0$ (i.e., $\frac{E_{b}}{N_{0}} \rightarrow \infty$ ) the density is given by:

$$
\lambda_{\epsilon}^{T}(b)=\kappa b\left(2^{b}-1\right)^{-\frac{2}{\alpha}} .
$$

In this limiting regime, a closed-form solution for $b^{*}$ can be reached.

Theorem 2: The optimum operating spectral efficiency $b^{*}$ in the absence of thermal noise $\left(N_{0}=0 \leftrightarrow \frac{E_{b}}{N_{0}}=\infty\right)$ is the unique solution to:

$$
b^{*}=\left(\log _{2} e\right) \frac{\alpha}{2}\left(1-2^{-b^{*}}\right),
$$

which can be written in closed form as:

$$
b^{*}=\log _{2} e\left[\frac{\alpha}{2}+\mathcal{W}\left(-\frac{\alpha}{2} e^{-\frac{\alpha}{2}}\right)\right]
$$

where $\mathcal{W}(z)$ is the principle branch of the Lambert $\mathcal{W}$ function and thus solves $\mathcal{W}(z) e^{\mathcal{W}(z)}=z$.

Proof: The result can be shown by directly maximizing (15) or by solving the fixed point equation given in Theorem 1 while keeping only the $\frac{E_{b}}{N_{0}}$ terms. The latter approach yields:

$$
\frac{2}{\alpha} b^{2} 2^{b} \log _{e} 2-b\left(2^{b}-1\right)=0,
$$

which is easily manipulated into the form of (16). To get (17) we manipulate (16) into the form $\left(b^{*} \log _{e} 2-\frac{\alpha}{2}\right) e^{b^{*}} \log _{e} 2=$ $-\frac{\alpha}{2}$. Multiplying both sides by $e^{-\frac{\alpha}{2}}$ yields $\left(b^{*} \log _{e} 2-\frac{\alpha}{2}\right) e^{b^{*} \log _{e} 2-\frac{\alpha}{2}}=-\frac{\alpha}{2} e^{-\frac{\alpha}{2}}$, from which we have $\mathcal{W}\left(-\frac{\alpha}{2} e^{-\frac{\alpha}{2}}\right)=$ $b^{*} \log _{e} 2-\frac{\alpha}{2}$ and thus the result.

The optimum depends only on the path loss exponent $\alpha$, and it is straightforward to show that $b^{*}$ is an increasing function of $\alpha, b^{*}$ is upper bounded by $\frac{\alpha}{2} \log _{2} e$, and that $b^{*} /\left(\frac{\alpha}{2} \log _{2} e\right)$ converges to 1 as $\alpha \rightarrow \infty$. In Fig. 3 the optimal spectral efficiency $b^{*}$ and $\frac{\lambda_{\epsilon}^{T}\left(b^{*}\right)}{\kappa}$ are plotted versus path-loss exponent $\alpha$. The optimal spectral efficiency is very small for $\alpha$ close to 2 but then increases nearly linearly with $\alpha$; for example, the optimal spectral efficiency for $\alpha=3$ is $1.26 \mathrm{bps} / \mathrm{Hz}(\beta=1.45 \mathrm{~dB})$. Note the non-monotonic behavior of $\frac{\lambda_{\epsilon}^{T}\left(b^{*}\right)}{\kappa}$ with $\alpha$ : the minimum occurs at $\alpha=2.77$, where $\frac{\lambda_{\epsilon}^{T}\left(b^{*}\right)}{\kappa}=b^{*}=1$. 


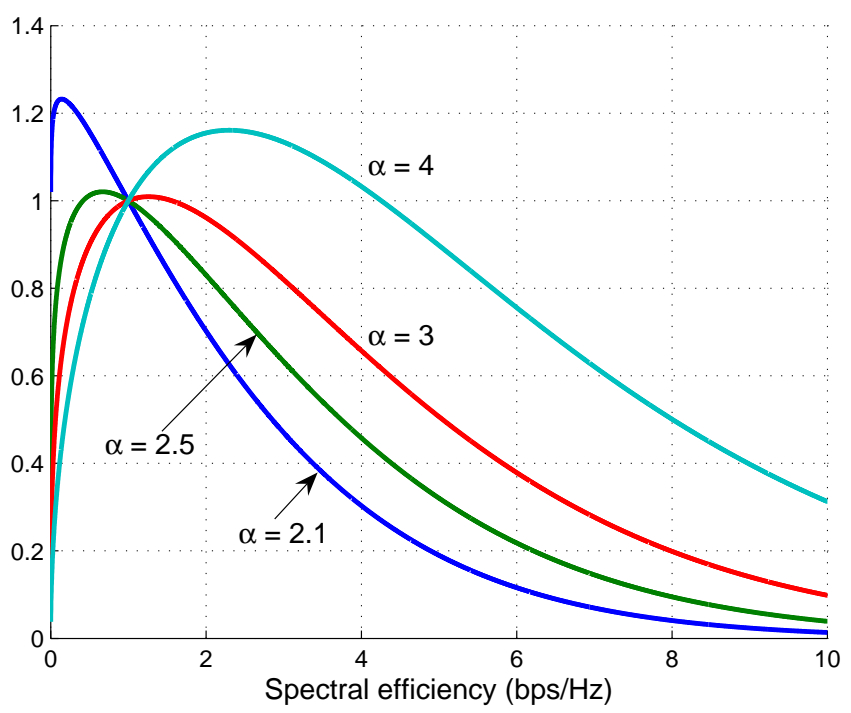

Fig. 4. Density Constant $\frac{\lambda_{\epsilon}^{T}(b)}{\kappa}$ vs. Spectral Efficiency $b$ for Interference-Limited Networks, $\alpha=2.1,2.5,3,4$.

To gain an intuitive understanding of the optimal solution, let us first consider the behavior of $\lambda_{\epsilon}^{T}(b)$ when $b$ is small, i.e. $b \ll 1$. Because $e^{x}-1 \approx x$ for small $x$, the SINR threshold increasing approximately linearly with $b$ : $\beta=2^{b}-1 \approx b \log _{e} 2$. Plugging into (15) yields:

$$
\lambda_{\epsilon}^{T}(b)=\kappa b\left(2^{b}-1\right)^{-\frac{2}{\alpha}} \approx \kappa b \cdot b^{-\frac{2}{\alpha}}=\kappa b^{\left(1-\frac{2}{\alpha}\right)} .
$$

For any path-loss exponent $\alpha>2$, the density of transmissions increases as $b^{\left(1-\frac{2}{\alpha}\right)}$. Therefore, increasing the number of sub-bands $N$, or equivalently increasing the spectral efficiency $b$, leads to an increased transmission capacity, as long as the linear approximation to $\beta$ remains valid. Recall that the area consumed by each transmission is proportional to $\beta^{\frac{2}{\alpha}}$ (equation 7): if $\beta \sim b$, then area increases sub-linearly as $b^{\frac{2}{\alpha}}$ and this increase is offset by the linear increase in the number of parallel transmissions. When $b$ becomes larger, $\beta$ begins to grow exponentially rather than linearly with $b$ (i.e., SINR must be doubled in $d B$ units rather than in linear units in order to double spectral efficiency) and thus the benefit of further increasing the number of sub-bands is far outweighed by the SINR/area increase.

This behavior is illustrated in Fig. 4, where $\lambda_{\epsilon}^{T}(b)=\kappa b\left(2^{b}-1\right)^{-\frac{2}{\alpha}}$ (with $\kappa=1$ ) is plotted versus $b$ for different values of $\alpha$. The function increases rapidly when $b$ is small, but then decreases rapidly beyond its peak when the SINR cost becomes prohibitive. A larger path loss exponent makes the system less sensitive to interference, and thus the peak is attained at a larger value of $b$. It is interesting to note that all of the curves intersect at $b=1$ because $\lambda_{\epsilon}^{T}(1)=\kappa$ for any value of $\alpha$. Although $b=1$ is quite sub-optimal when $\alpha$ is near $2, \kappa$ is reasonably close to the optimal $\kappa b^{*}\left(2^{b^{*}}-1\right)^{-\frac{2}{\alpha}}$ for exponents between 2.5 and 5 and thus is a rather robust operating point if the path loss exponent is not known exactly.

A Design Example. Consider wireless LAN parameters that are conceptually similar to those of an $2.4 \mathrm{GHz}$ 802.11 system, that uses $N=3$ bands of about $20 \mathrm{MHz}$. Assume the usable bandwidth is a total of $W=60 \mathrm{MHz}$, and that the desired rate is $R=10 \mathrm{Mbps}$ and $\alpha=3$. From Theorem 2 we can determine that

$$
N^{*}=\frac{b^{*}}{R / W}=\frac{1.26}{R / W}=7.56,
$$

so the optimum partition is about $N^{*}=8$, or bands of $7.5 \mathrm{MHz}$. If however the data rate requirement is higher, like $60 \mathrm{Mbps}$, then it can quickly be confirmed that $N^{*}=1$. That is, the maximum number of users can be accommodated at the higher data rate if each of them uses the entire band, since they can accept a lower received SINR with such a large bandwidth. 


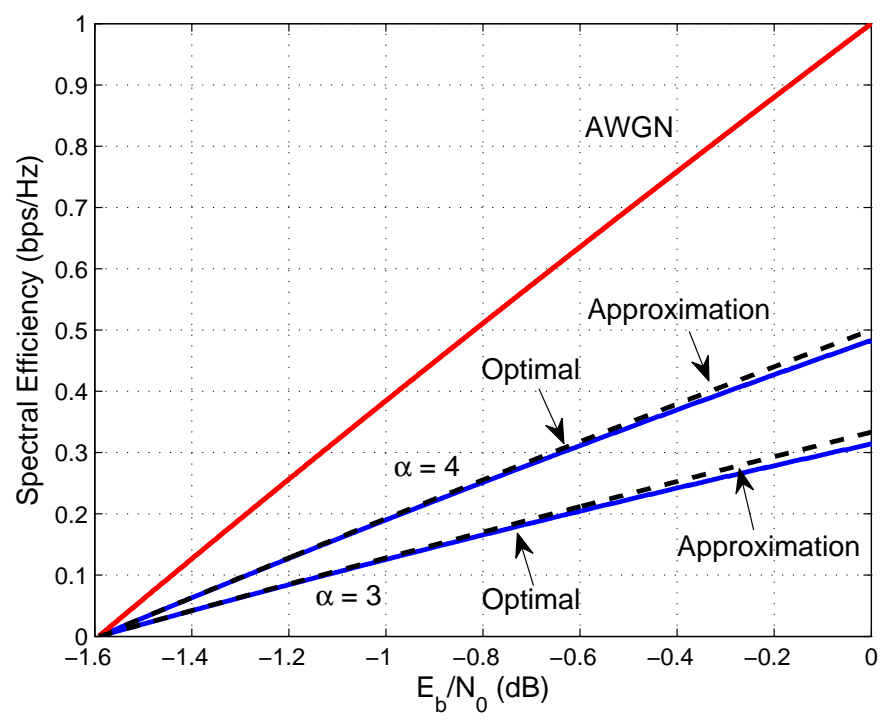

Fig. 5. Optimal Spectral Efficiency $b^{*}$ vs. $\frac{E_{b}}{N_{0}}$ for Power-Limited Networks, $\alpha=3,4$.

\section{PARTitioning For POWER-Limited Networks}

In the power-limited regime where $\frac{E_{b}}{N_{0}}$ is close to $-1.59 \mathrm{~dB}$, we can obtain a simple characterization of $b^{*}$ that is accurate up to a quadratic term by solving the fixed point equation given in Theorem 1 ;

Theorem 3: The optimum operating spectral efficiency $b^{*}$ in the power-limited regime $\left(\frac{E_{b}}{N_{0}}\right.$ slightly larger than $-1.59 \mathrm{~dB}$ ) is given by:

$$
b^{*}=\left(1-\frac{2}{\alpha}\right) C\left(\frac{E_{b}}{N_{0}}\right)+O\left(b^{2}\right)
$$

where $C\left(\frac{E_{b}}{N_{0}}\right)$ is the AWGN spectral efficiency at $\frac{E_{b}}{N_{0}}$ as defined in (12).

Furthermore, the density in the wideband regime is characterized as:

$$
\frac{\lambda_{\epsilon}^{T}\left(b^{*}\right)}{\kappa}=\left((1-\delta)^{(1-\delta)} \delta^{\delta} 2^{-\delta}\right) C\left(\frac{E_{b}}{N_{0}}\right)+O\left(b^{2}\right)
$$

where $\delta \triangleq \frac{2}{\alpha}$ and $(1-\delta)^{(1-\delta)} \delta^{\delta} 2^{-\delta}<1$ for all $\alpha>2$.

Proof: See Appendix [1]

Fig. 5 contains plots of the numerically computed $b^{*}$, the approximation $\left(1-\frac{2}{\alpha}\right) \mathrm{C}\left(\frac{E_{b}}{N_{0}}\right)$, and $\mathrm{C}\left(\frac{E_{b}}{N_{0}}\right)$ versus $\frac{E_{b}}{N_{0}}$ for $\alpha=3$ and $\alpha=4$. Fig. 6 contains plots of the numerically computed $\frac{\lambda_{\epsilon}^{T}\left(b^{*}\right)}{\kappa}$, the approximation from (20), and $\mathrm{C}\left(\frac{E_{b}}{N_{0}}\right)$ versus $\frac{E_{b}}{N_{0}}$ for $\alpha=2.01$ and $\alpha=3$ (the curve for $\alpha=4$ is nearly indistinguishable from $\alpha=3$ ). Both approximations are seen to be very accurate.

Although intuition might suggest that interference can be ignored when thermal noise is so large, this is not the case. If $b$ is chosen only slightly smaller than $C\left(\frac{E_{b}}{N_{0}}\right)$, the SINR threshold is almost equal to the interference-free SNR and thus each receiver is extremely sensitive to interference. As a result each communication consumes a large area, and this offsets the bandwidth savings of using a large $b$. On the other extreme, small $b$ corresponds to a small area because the SINR threshold is much smaller than the interference-free SNR, but this is offset by a large bandwidth which causes $\lambda_{\epsilon}^{T}(b) \rightarrow 0$ as $b \rightarrow 0$.

This behavior is illustrated in Fig. 7, where $\lambda_{\epsilon}^{T}(b)$ (with $\kappa=1$ ) is plotted versus $b$ for $\alpha=2.2,3$, and 4 at $\frac{E_{b}}{N_{0}}=-0.82 \mathrm{~dB}$ (for which $\mathrm{C}\left(\frac{E_{b}}{N_{0}}\right)=0.5 \mathrm{bps} / \mathrm{Hz}$ ). Choosing $b$ near either extreme leads to very poor performance for any $\alpha$. Notice that all three curves intersect when $b$ satisfies $\frac{1}{2^{b}-1}-\frac{1}{b \frac{E_{b}}{N_{0}}}=1$. This condition is satisfied when the SINR threshold is equal to $\frac{\mathrm{SNR}}{1+\mathrm{SNR}}$, where SNR is the interference-free SNR, and thus $b=\log _{2}\left(1+\frac{\mathrm{SNR}}{1+\mathrm{SNR}}\right)$. 


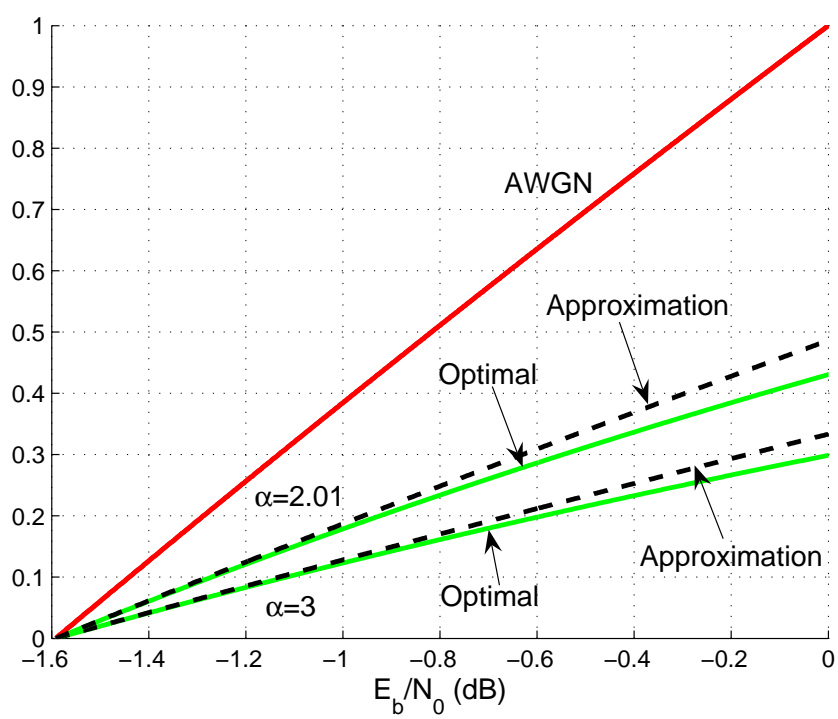

Fig. 6. Optimal Density Constant $\frac{\lambda_{\epsilon}^{T}\left(b^{*}\right)}{\kappa}$ vs. $\frac{E_{b}}{N_{0}}$ for Power-Limited Networks, $\alpha=2.01,3$.

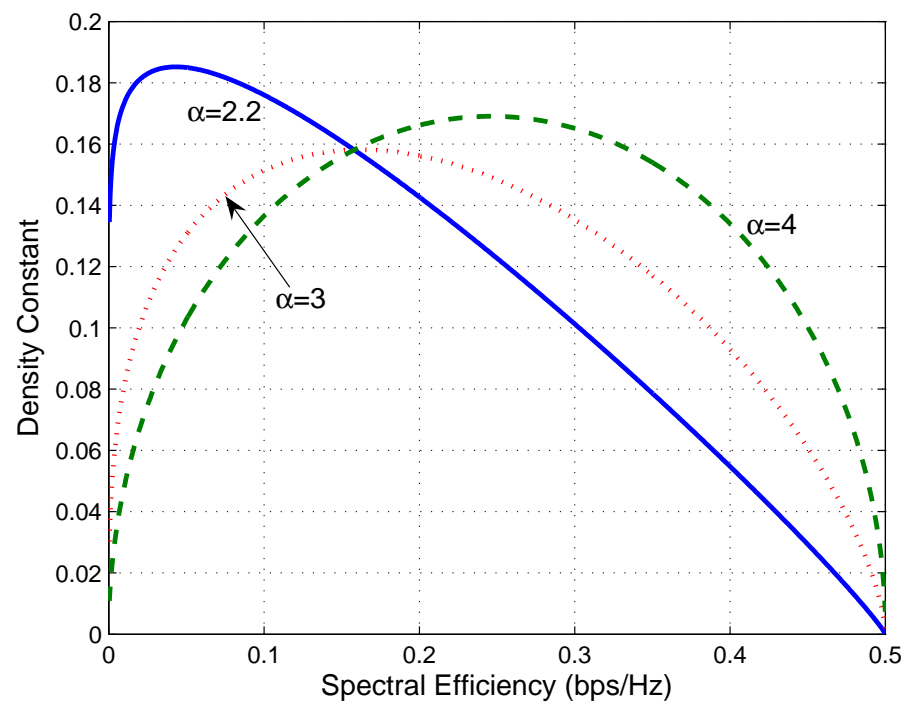

Fig. 7. Density Constant $\frac{\lambda_{\epsilon}^{T}(b)}{\kappa}$ vs. Spectral Efficiency $b$ for Power-Limited Networks.

By a simple calculation using tools from [3], the intersection point corresponds to $b=\frac{1}{3} \mathrm{C}\left(\frac{E_{b}}{N_{0}}\right)$. Although this choice of spectral efficiency is only optimal for $\alpha=3$, it is quite close to optimal for path loss exponents that are not too near 2 and thus is a robust operating point in the power-limited regime, analogous to the choice $b=1$ in the interference-limited regime.

Finally, note that multi-user interference decreases the marginal benefit of increased power (i.e., $\frac{E_{b}}{N_{0}}$ ) as compared to an interference-free channel. The analogous quantity for the spatial network considered here is the area spectral efficiency (ASE), which is computed relative to the total bandwidth of $W \mathrm{~Hz}$ and is equal to $\lambda_{\epsilon}^{T}\left(b^{*}\right)\left(\frac{R}{W}\right) \mathrm{bps} / \mathrm{Hz}$ per $m^{2}$. In an AWGN channel, spectral efficiency increases at a slope of $2 \mathrm{bps} / \mathrm{Hz}$ per $3 \mathrm{~dB}$ in the wideband regime $\left(\mathcal{S}_{0}=2\right)$ [3], while (20) implies that ASE increases only at a rate of $2^{1-\delta}\left((1-\delta)^{(1-\delta)} \delta^{\delta}\right)(<2)$ bps/Hz per $3 \mathrm{~dB}$.

\section{NUMERICAL RESUlTS AND EXTENSIONS}

In the following we present numerical results to illustrate the value of bandwidth partitioning. With system parameters chosen as $\epsilon=0.1, N_{0}=10^{-6}, \alpha=4, d=10, R=1 \mathrm{Mbps}$, and $W=10 \mathrm{MHz}$, the total density 


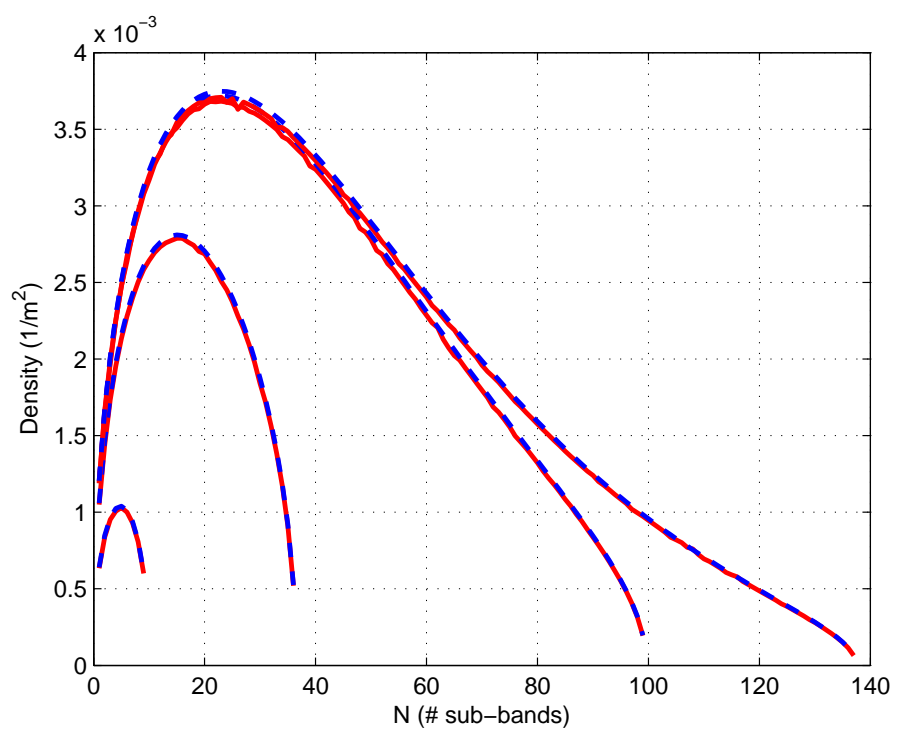

Fig. 8. Numerically computed $\lambda_{\epsilon}^{T}(N)$ versus $N$ for $\alpha=4$ and $\frac{E_{b}}{N_{0}}=30,20,5$, and $0 \mathrm{~dB}$ (top to bottom). Solid and dotted lines were computed using (5) and pure Monte Carlo simulation, respectively.

$\lambda_{\epsilon}^{T}(N)$ is computed via full Monte Carlo simulation (of outage probability at different densities) and with equation (5) using the numerically computed value $F_{Z}^{-1}(0.1)=0.1015$. Fig. 8 contains plots of both quantities for $\frac{E_{b}}{N_{0}}$ equal to $30,20,5$, and $0 \mathrm{~dB}$, and the curves match almost exactly with any difference due purely to simulation error. According to the chosen parameters we have $N^{*}=\frac{b^{*}}{R / W}=10 b^{*}$ and $\kappa=0.0032$. Note that the optimizing spectral efficiency $b^{*}$ and the value of $\frac{\lambda_{\epsilon}^{T}\left(b^{*}\right)}{\kappa}$ can be read from Fig. 1. The top two set of curves are for $\frac{E_{b}}{N_{0}}=30 \mathrm{~dB}$ and $\frac{E_{b}}{N_{0}}=20 \mathrm{~dB}$, both of which correspond to the interference-limited regime where $b^{*}=2.3 \mathrm{bps} / \mathrm{Hz}\left(N^{*}=23\right)$. The curves are nearly indistinguishable near the optimal $N^{*}$ because performance is essentially independent of $\frac{E_{b}}{N_{0}}$ in the interference-limited regime. The middle set of curves correspond to $\rho=\frac{E_{b}}{N_{0}}=5 \mathrm{~dB}$, which is between the two extremes. At this point $b^{*}=1.5 \mathrm{bps} / \mathrm{Hz}\left(N^{*}=15\right)$ and $\frac{\lambda_{\epsilon}^{T}\left(b^{*}\right)}{k}=0.8$; reducing power by $15 \mathrm{~dB}$ while keeping all other parameters fixed reduces density/ASE by approximately a third. The bottom curves correspond to $\rho=\frac{E_{b}}{N_{0}}=0$ $\mathrm{dB}$, which is in the wideband regime. At this point $\mathrm{C}\left(\frac{E_{b}}{N_{0}}\right)=1$ and $b^{*}=\left(1-\frac{\alpha}{2}\right) C\left(\frac{E_{b}}{N_{0}}\right)=0.5 \mathrm{bps} / \mathrm{Hz}\left(N^{*}=5\right)$, and the area spectral efficiency is reduced to 0.1.

While $\frac{E_{b}}{N_{0}}$ is generally thought to be adjusted by varying transmit power, it can also be adjusted by fixing the power and varying the rate $R$. The area spectral efficiency $\lambda_{\epsilon}^{T}\left(b^{*}\right) \frac{R}{W} \mathrm{bps} / \mathrm{Hz} / \mathrm{m}^{2}$ is equal to $\left(\frac{-\log _{e}(1-\epsilon)}{\pi d^{2}}\right) b^{*}\left(2^{b^{*}}-1\right)^{-\frac{2}{\alpha}}$, and therefore depends only on $\frac{E_{b}}{N_{0}}$ but not on the particular values of $R$ and $\rho$. As a result, if a network is operating outside of the interference-limited regime, ASE can be tremendously increased by either increasing power or decreasing rate (while keeping power fixed). However, this is only possible until the interference-limited regime is reached; once there, ASE is unaffected by $\frac{E_{b}}{N_{0}}$.

\section{A. Direct Sequence Spread-Spectrum}

Direct-sequence (DS) spread spectrum is a well-established method for spectrum sharing in wireless networks. If DS is used with a spreading factor of $N$, a signal with an information bandwidth (i.e., symbol rate) of $\frac{W}{N} \mathrm{~Hz}$ can be spread across the entire system bandwidth of $W \mathrm{~Hz}$. This is quite different than the method investigated so far, which be thought of as either FDMA or slow frequency-hopping. In [17] it is shown that DS is significantly inferior to splitting the frequency band (FDMA) for any particular bandwidth partition because it is preferable to avoid interference (FDMA) rather than to suppress it (DS), and this conclusion also holds if the bandwidth is optimally partitioned.

If direct sequence is used with completely separate despreading and decoding (assuming spreading suppresses 
interference by a factor of $N$ ), the SINR after despreading is given by:

$$
\operatorname{SINR}_{\mathrm{DS}}=\frac{\rho d^{-\alpha}}{N_{0}(W / N)+\frac{1}{N} \sum_{i \in \Pi(\lambda)} \rho X_{i}^{-\alpha}} .
$$

With some simple manipulation the outage probability is given by:

$$
\mathbb{P}\left[\operatorname{SINR}_{\mathrm{DS}} \leq \beta(N)\right]=\mathbb{P}\left[\frac{\rho d^{-\alpha}}{N_{0} W+\sum_{i \in \Pi(\lambda)} \rho X_{i}^{-\alpha}} \leq \frac{\beta(N)}{N}\right]
$$

where $\beta(N)$ is defined in (4). Therefore, the total transmission density for DS with spreading factor $N$ is equal to $\lambda_{\epsilon}$ as defined in (2) with threshold $\frac{\beta(N)}{N}$ and $\eta=N_{0} W$. However, $\frac{\beta(N)}{N}$ is an increasing function of $N$ and thus total density monotonically decreases with $N$ if DS is used. Direct-sequence increases SINR by at most a factor of $N$, but this gain is offset by the fact that the SINR threshold increases at least linearly with $N$. As a result a DS system performs no better than an FDMA/FH system with $N=1$, which corresponds to $\lambda_{\epsilon}^{T}(1)$ in (5) and which is generally much smaller than the optimal $\lambda_{\epsilon}^{T}\left(N^{*}\right)$. Although DS has strengths unrelated to spectral efficiency, such as security and MAC design [18], these benefits come at a significant performance penalty.

\section{B. Effect of Frequency-Flat Fading and Variable TX-RX Distances}

In the presence of fading and variable distances, the SINR expression becomes:

$$
\operatorname{SINR}_{0}=\frac{\rho d^{-\alpha} h_{0}}{\eta+\sum_{i \in \Pi(\lambda)} \rho X_{i}^{-\alpha} h_{i}}
$$

where $h_{i}$ denotes the power of the fading coefficient from TX $i$ to the reference receiver, $h_{0}, h_{1}, \ldots$ are chosen iid according to some distribution $F_{H}$, and $d$ is a random variable chosen according to distribution $F_{D}$. If we define $Z \triangleq\left(\sum_{i \in \Pi(1 / \pi)} h_{i} Z_{i}^{-\alpha}\right)^{-\frac{2}{\alpha}}$, and $G=d^{-\alpha} h_{0}$, then simple manipulation yields:

$$
\begin{aligned}
P_{\text {out }}(\lambda, \beta, \eta) & =\mathbb{P}\left(Z^{-\frac{\alpha}{2}} \geq(\pi \lambda)^{-\frac{\alpha}{2}}\left(\frac{G}{\beta}-\frac{\eta}{\rho}\right)\right) \\
& =\mathbb{P}\left(G \leq \frac{\beta \eta}{\rho}\right)+\mathbb{P}\left(Z \leq \pi \lambda\left(\frac{G}{\beta}-\frac{\eta}{\rho}\right)^{-\frac{2}{\alpha}} \mid G \geq \frac{\beta \eta}{\rho}\right) \mathbb{P}\left(G \geq \frac{\beta \eta}{\rho}\right)
\end{aligned}
$$

The first term is the probability of an outage due to insufficient received signal power, i.e., $G$ is so small that the interference-free SNR is below the SINR threshold, while the second is the probability of outage conditioned on sufficient signal power. Because of the somewhat involved expression for outage probability, it is more convenient to consider bandwidth partitioning in terms of outage minimization rather than density maximization. In the purely interference-limited regime $\left(N_{0}=0\right)$, the first term in (23) disappears and the outage probability (in terms of $N$ ) is given by:

$$
P_{\text {out }}(N)=\mathbb{P}\left(Z G^{\frac{2}{\alpha}} \leq \pi\left(\frac{\lambda}{N}\right)\left(2^{\frac{N R}{W}}-1\right)^{\frac{2}{\alpha}}\right)=\mathbb{P}\left(Z G^{\frac{2}{\alpha}} \leq \pi \lambda\left(\frac{R}{W}\right) \frac{1}{b}\left(2^{b}-1\right)^{\frac{2}{\alpha}}\right),
$$

where we have again used $b=\frac{N R}{W}$. Outage is minimized by minimizing $\frac{1}{b}\left(2^{b}-1\right)^{\frac{2}{\alpha}}$, which is clearly equivalent to the problem solved in Section [V] Thus, the interference-limited solution given in Theorem 2 is also optimal in the presence of fading and variable distances.

However, the same is not true when there is positive noise power. By substituting the appropriate values into (23) and manipulating the second addend, outage is characterized as:

$$
\begin{aligned}
P_{\mathrm{out}}(N) & =\mathbb{P}\left(G \leq g^{*}\right)+\int_{g^{*}}^{\infty} F_{Z}\left(\pi \frac{\lambda}{N} x^{-\frac{2}{\alpha}}\left(\frac{1}{2^{\frac{N R}{W}}-1}-\frac{N_{0} W}{N \rho x}\right)^{-\frac{2}{\alpha}}\right) f_{G}(x) d x \\
& =\mathbb{P}\left(G \leq g^{*}\right)+\int_{g^{*}}^{\infty} F_{Z}\left(\pi \lambda \frac{R}{W} x^{-\frac{2}{\alpha}} \frac{1}{b}\left(\frac{1}{2^{b}-1}-\frac{N_{0} R}{b \rho x}\right)^{-\frac{2}{\alpha}}\right) f_{G}(x) d x
\end{aligned}
$$




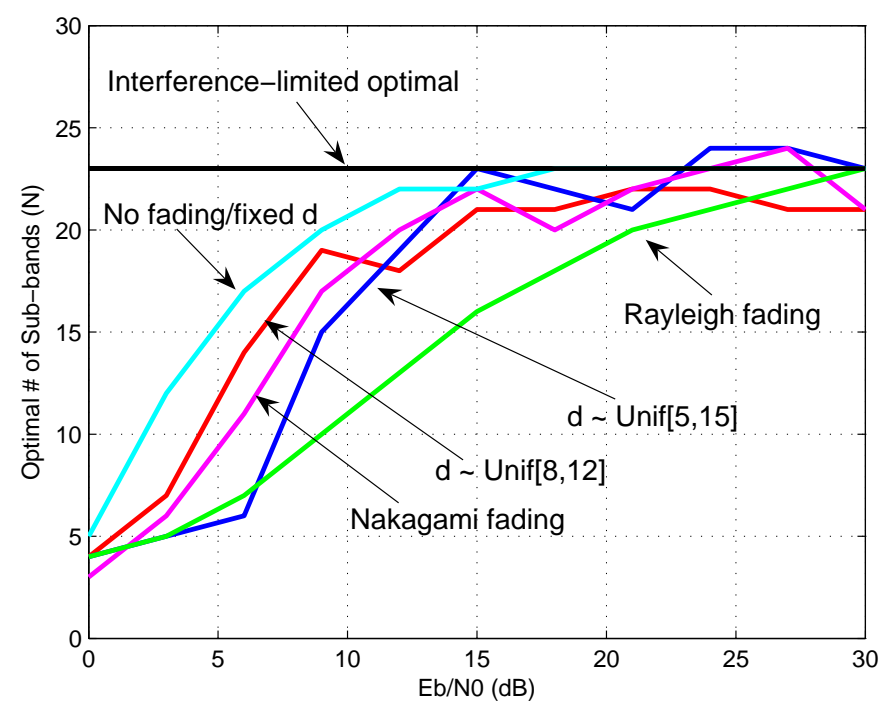

Fig. 9. Optimal Value of $N$ vs. $\frac{E_{b}}{N_{0}}$

where $g^{*}=\left(2^{\frac{N R}{W}}-1\right)\left(\frac{N_{0} W}{N \rho}\right)=\left(\frac{2^{b}-1}{b}\right)\left(\frac{N_{0} R}{\rho}\right)$. The first term, which represents outage due to insufficient received power, increases with $N$ because $g^{*}$ is an increasing function of $N$. The integrand in the second term is the outage probability conditioned on $G=x$, and is precisely of the form investigated earlier with $\frac{E_{b}}{N_{0}}=\frac{\rho x}{N_{0} R}$. Therefore, Theorem 1 characterizes the value of $N$ that minimizes the integrand for each value of $x$, but does not generally characterize the minimizer of (24). However, the solution from Theorem 1 does become increasingly accurate as transmission power is increased (i.e., the interference-limited regime is approached) and as the variation in the fading and TX-RX distances decreases. Increasing power causes the first term in (24) to decrease and eventually become negligible, while decreasing variation in $G$ reduces variation in the effective energy per bit $\frac{E_{b}}{N_{0}}=\frac{\rho x}{N_{0} R}$.

To illustrate this, Fig. 9 displays the outage minimizing value of $N$ (computed via Monte Carlo) versus $\frac{E_{b}}{N_{0}}$ for four different settings: Rayleigh fading and Nakagami fading $(m=5)$ for fixed $d=10$, and no fading and variable distances for $d$ uniform in $[8,12]$ and $[5,15]$. The relevant parameters are: $W=5 \mathrm{MHz}, N_{0}=10^{-6}, R=1 \mathrm{Mbps}$, $\lambda=\frac{.01}{\pi} \mathrm{m}^{-2}, \alpha=4$. The jitter in the curves is due to simulation error. For sufficiently large $\frac{E_{b}}{N_{0}}$, the optimal does indeed converge to the optimal value for a purely interference-limited $\left(N_{0}=0\right)$ network. Furthermore, the optimizing $N$ tends towards the Theorem 1 solution for more benign fading (Nakagami) and for smaller distance variation 4 Based on (24), the truly optimal $N$ seems to depend on the particular fading and distance distributions and appears somewhat intractable; further investigation is left for future work.

\section{CONCLUSION}

In this work we studied the problem of bandwidth partitioning in a decentralized network and derived the optimal operating spectral efficiency, assuming multi-user interference is treated as noise and no transmission scheduling is performed. A network can operate at this optimal point by dividing the total bandwidth into sub-bands sized such that each transmission occurs on one of the sub-bands at precisely the optimal spectral efficiency.

The essence of this problem is determining the optimum balance between the time-frequency resources and area resources consumed by each transmission. Using many time-frequency resources to transmit a finite number of bits corresponds to operating at a low spectral efficiency. This translates to a small required SINR, and thus only a small area must be free of interfering transmissions. On the other hand, using few time-frequency resources corresponds to a large spectral efficiency and in turn a large SINR and interferer-free area. Our analysis shows that the optimal

\footnotetext{
${ }^{4}$ Our recent work has shown that there can be a substantial benefit to reducing variation in received signal power by adjusting transmit power to partially compensate for reduced signal power [19]; thus, systems with relatively small signal power variation are particularly relevant.
} 
depends only on the path loss exponent and energy per information bit. If thermal noise is negligible the optimal spectral efficiency lies between the low- and high-SNR extremes, while in the power-limited regime the optimal is a fraction of the maximum possible spectral efficiency in the absence of interference. Furthermore, the optimal spectral efficiency is always an increasing function of the path loss exponent and of the energy per information bit.

\section{APPENDIX I \\ PROOF OF THEOREM 1}

In order to prove the result it is convenient to work with natural logarithms:

$$
\lambda_{\epsilon}^{T}(b)=\kappa b\left(\frac{1}{2^{b}-1}-\frac{1}{b} \frac{1}{\frac{E_{b}}{N_{0}}}\right)^{\frac{2}{\alpha}}=\left(\frac{\kappa}{\log _{e} 2}\right) n\left(\frac{1}{\mathrm{e}^{n}-1}-\frac{1}{n} \frac{1}{\tilde{\mathcal{E}}}\right)^{\frac{2}{\alpha}}=\lambda_{\epsilon}^{T}(n)
$$

where $n \triangleq b \log _{e} 2$ and $\tilde{\mathcal{E}} \triangleq \frac{\frac{E_{b}}{N_{0}}}{\log _{e} 2}$. Ignoring constant $\frac{\kappa}{\log _{e} 2}$ and defining $\delta=\frac{2}{\alpha}$, the first derivative is:

$$
\begin{aligned}
\frac{d}{d n}\left[\lambda_{\epsilon}^{T}(n)\right] & =\left(\frac{1}{\mathrm{e}^{n}-1}-\frac{1}{\tilde{\mathcal{E}} n}\right)^{\delta}+n \delta\left(\frac{1}{\mathrm{e}^{n}-1}-\frac{1}{\tilde{\mathcal{E}} n}\right)^{\delta-1}\left(\frac{-\mathrm{e}^{n}}{\left(\mathrm{e}^{n}-1\right)^{2}}+\frac{1}{\tilde{\mathcal{E}} n^{2}}\right) \\
& =\frac{\left(\frac{1}{\mathrm{e}^{n}-1}-\frac{1}{\tilde{\mathcal{E}} n}\right)^{\delta-1}}{\tilde{\mathcal{E}} n\left(\mathrm{e}^{n}-1\right)^{2}}\left[\tilde{\mathcal{E}} n\left(\mathrm{e}^{n}-1\right)-\tilde{\mathcal{E}} \delta n^{2} \mathrm{e}^{n}-(1-\delta)\left(\mathrm{e}^{n}-1\right)^{2}\right] .
\end{aligned}
$$

Because the first term is positive for any $n>0$, the derivative is equal to zero if and only if:

$$
\tilde{\mathcal{E}} n\left(\mathrm{e}^{n}-1\right)-\tilde{\mathcal{E}} \delta n^{2} \mathrm{e}^{n}-(1-\delta)\left(\mathrm{e}^{n}-1\right)^{2}=0 .
$$

Substituting $n=b \log _{e} 2$ and $\tilde{\mathcal{E}}=\frac{\frac{E_{b}}{N_{0}}}{\log _{e} 2}$ yields the fixed point equation in (13). Although $\lambda_{\epsilon}^{T}(n)$ is neither convex nor concave, we can show it has a unique maximizer at the unique positive solution to the above equation. It is easy to check that $\lambda_{\epsilon}^{T}(0)=\lambda_{\epsilon}^{T}\left(n_{\max }\right)=0$ and $\lambda_{\epsilon}^{T}(n)>0$ for $0<n<n_{\max }$, where $n_{\max }=\mathrm{C}\left(\frac{E_{b}}{N_{0}}\right) \log _{e} 2$. Therefore the function is maximized at a point where its derivative is zero. Furthermore, (28) is satisfied at any point where the derivative is zero and thus (28) must have at least one positive solution. To show that (28) has a unique positive solution, define $\nu(n)=\tilde{\mathcal{E}} \delta n^{2} \mathrm{e}^{n}+(1-\delta)\left(\mathrm{e}^{n}-1\right)^{2}$ and $\nu(n)=\tilde{\mathcal{E}} n\left(\mathrm{e}^{n}-1\right)$. Equation (28) is satisfied if and only if $\mu(n)=\nu(n)$. Note that $\mu(0)=\nu(0)=0$ and $\mu(n)>0$ and $\nu(n)>0$ for all $n>0$. Simple calculations show that each function is strictly convex. Hence $\mu(n), \nu(n)$ are positive valued, non-decreasing, strictly convex functions, and based on this it is straightforward to argue that there is at most one positive solution of $\mu(n)=\nu(n)$.

To show $n^{*}(\tilde{\mathcal{E}}, \delta)$ increases with $\tilde{\mathcal{E}}$, define the LHS of $(28)$ as $f(n, \tilde{\mathcal{E}}, \delta)$. By the properties shown earlier, $f(n, \tilde{\mathcal{E}}, \delta)>0$ for $0<n<n^{*}(\tilde{\mathcal{E}}, \delta)$ and $f(n, \tilde{\mathcal{E}}, \delta)<0$ for $n>n^{*}(\tilde{\mathcal{E}}, \delta)$. As a result, $n^{*}(\tilde{\mathcal{E}}, \alpha)$ increases with $\tilde{\mathcal{E}}$ if for any $\tilde{\mathcal{E}}^{\prime}>\tilde{\mathcal{E}}, f\left(n, \tilde{\mathcal{E}}^{\prime}, \delta\right)>0$ for $0<n<n^{*}(\tilde{\mathcal{E}}, \delta)$. To prove this property, choose any $n, \tilde{\mathcal{E}}$ such that $f(n, \tilde{\mathcal{E}}, \delta)=\tilde{\mathcal{E}}\left(n\left(\mathrm{e}^{n}-1\right)-\delta n^{2} \mathrm{e}^{n}\right)-(1-\delta)\left(\mathrm{e}^{n}-1\right)^{2}>0$. Since $(1-\delta)\left(\mathrm{e}^{n}-1\right)^{2}>0$ for any $n$, this implies $n\left(\mathrm{e}^{n}-1\right)-\delta n^{2} \mathrm{e}^{n}>0$. Thus, for any $\tilde{\mathcal{E}}^{\prime}>\tilde{\mathcal{E}}$ :

$$
f\left(n, \tilde{\mathcal{E}}^{\prime}, \delta\right)=\tilde{\mathcal{E}}^{\prime}\left(n\left(\mathrm{e}^{n}-1\right)-\delta n^{2} \mathrm{e}^{n}\right)-(1-\delta)\left(\mathrm{e}^{n}-1\right)^{2}>f(n, \tilde{\mathcal{E}}, \delta)>0 .
$$

By a similar argument, if $f(n, \tilde{\mathcal{E}}, \delta)$ is a decreasing function of $\delta$ then $n^{*}(\tilde{\mathcal{E}}, \delta)$ decreases with $\delta$, i.e., increases with $\alpha$. To prove this, note that the partial of $f(n, \tilde{\mathcal{E}}, \delta)$ with respect to $\delta$ is $\left(\mathrm{e}^{n}-1\right)^{2}-\tilde{\mathcal{E}} n^{2} \mathrm{e}^{n}$. Recall that $n \leq n^{\max }$ is equivalent to $\tilde{\mathcal{E}} n \geq \mathrm{e}^{n}-1$. This allows:

$$
\tilde{\mathcal{E}} n^{2} \mathrm{e}^{n}-\left(\mathrm{e}^{n}-1\right)^{2} \geq\left(\mathrm{e}^{n}-1\right) n \mathrm{e}^{n}-\left(\mathrm{e}^{n}-1\right)^{2}=\left(\mathrm{e}^{n}-1\right)\left(n \mathrm{e}^{n}-\mathrm{e}^{n}+1\right) \geq 0 .
$$

The last expression is nonnegative on account of the fact that the function $n \mathrm{e}^{n}-\left(\mathrm{e}^{n}-1\right)$ has derivative $n \mathrm{e}^{n} \geq 0$. Thus $f(n, \tilde{\mathcal{E}}, \delta)$ is decreasing in $\delta$, i.e., increasing in $\alpha$. 


\section{APPENDIX II \\ PROOF OF THEOREM 3}

For convenience, we again work with the function in natural $\log$ form (see Appendix 【). To prove the result, we expand the exponential terms (using $\mathrm{e}^{x}=\sum_{k=0}^{\infty} \frac{x^{k}}{k !}$ ) in (28) to give:

$$
\tilde{\mathcal{E}} \delta n^{2}\left(1+n+O\left(n^{2}\right)\right)-\tilde{\mathcal{E}} n\left(n+\frac{n^{2}}{2}+O\left(n^{3}\right)\right)+(1-\delta)\left(n^{2}+n^{3}+O\left(n^{4}\right)\right)^{2}=0
$$

Cancelling a factor of $n^{2}$ throughout yields

$$
\tilde{\mathcal{E}} \delta\left(1+n+O\left(n^{2}\right)\right)-\tilde{\mathcal{E}}\left(1+\frac{1}{2} n+O\left(n^{2}\right)\right)+(1-\delta)\left(1+n+O\left(n^{2}\right)\right)=0
$$

which can be solved to yield a solution that is accurate to within a quadratic term:

$$
n^{*}=\frac{\tilde{\mathcal{E}}(1-\delta)+(\delta-1)}{\tilde{\mathcal{E}}\left(\delta-\frac{1}{2}\right)+(1-\delta)}+O\left(n^{2}\right) .
$$

We are interested in the behavior of $\frac{b^{*}}{\mathrm{C}\left(\frac{E_{b}}{N_{0}}\right)}$ as $\frac{E_{b}}{N_{0}} \rightarrow 0$ (or equivalently $\mathrm{C}\left(\frac{E_{b}}{N_{0}}\right) \rightarrow 0$ ). Because $n^{*}=b^{*} \log _{e} 2$ and $n_{\max }=\mathrm{C}\left(\frac{E_{b}}{N_{0}}\right) \log _{e} 2$ we can equivalently evaluate $\frac{n^{*}}{n_{\max }}$

$$
\lim _{n_{\max } \rightarrow 0}\left(\frac{n^{*}}{n_{\max }}\right)=\lim _{n_{\max } \rightarrow 0} \frac{1}{n_{\max }}\left(\frac{\tilde{\mathcal{E}}(1-\delta)+(\delta-1)}{\tilde{\mathcal{E}}\left(\delta-\frac{1}{2}\right)+(1-\delta)}\right) .
$$

By plugging in $\frac{\mathrm{e}^{n_{\max }-1}}{n_{\max }}=\tilde{\mathcal{E}}$ and using L'Hospital's rule, we can show the above limit is $1-\delta$, which implies $n^{*}=(1-\delta) n_{\max }+O\left(n^{2}\right)$, which in turn gives the final result:

$$
b^{*}=\left(1-\frac{2}{\alpha}\right) C\left(\frac{E_{b}}{N_{0}}\right)\left(\frac{E_{b}}{N_{0}}\right)+O\left(b^{2}\right) .
$$

Because our approximation is accurate within a quadratic, we have the following:

$$
\lim _{\mathrm{C}\left(\frac{E_{b}}{N_{0}}\right) \rightarrow 0} \frac{\lambda_{\epsilon}^{T}\left(b^{*}\right)}{\mathrm{C}\left(\frac{E_{b}}{N_{0}}\right)}=\lim _{\mathrm{C}\left(\frac{E_{b}}{N_{0}}\right) \rightarrow 0} \frac{\lambda_{\epsilon}^{T}\left((1-\delta) \mathrm{C}\left(\frac{E_{b}}{N_{0}}\right)\right)}{\mathrm{C}\left(\frac{E_{b}}{N_{0}}\right)} .
$$

By working with the natural $\log$ version of this equation and plugging in $\frac{\mathrm{e}^{n_{\max }-1}}{n_{\max }}=\tilde{\mathcal{E}}$, L'Hospital's rule can be used to show that this limit is equal to $\kappa(1-\delta)^{(1-\delta)} \delta^{\delta} 2^{-\delta}$, which yields (20).

\section{REFERENCES}

[1] N. Jindal, J. Andrews, and S. Weber, "Optimizing the SINR operating point of spatial networks," in Proceedings of Workshop on Information Theory and its Applications, San Diego, CA, Jan. 2007.

[2] —_ "Bandwidth-SINR tradeoffs in spatial networks," in Proceedings of IEEE Int. Symp. on Inform. Theory, June 2007.

[3] S. Verdu, "Spectral efficiency in the wideband regime," IEEE Trans. Inform. Theory, vol. 48, no. 6, pp. 1319-1343, June 2002.

[4] T. Rappaport, Wireless Communications: Principles \& Practice. Prentice Hall, 1996.

[5] J. G. Andrews, A. Ghosh, and R. Muhamed, Fundamentals of WiMAX. Prentice-Hall, 2007.

[6] K. L. Yeung and S. Nanda, "Channel management in microcell/macrocell cellular radio systems," IEEE Trans. on Veh. Technology, vol. 45, no. 4, pp. 601-12, Nov. 1996.

[7] M. Pursley and T. Royster, "Resource consumption in dynamic spectrum access networks: Applications and Shannon limits," in Proceedings of Workshop on Information Theory and its Applications, San Diego, CA, 2007.

[8] N. Ehsan and R. L. Cruz, "On the optimal SINR in random access networks with spatial reuse," in Proceedings of Conference on Information Sciences and Systems (CISS), 2006.

[9] M. Ebrahimi and A. Khandani, "Rate-constrained wireless networks with fading channels: Interference-limited and noise-limited regimes," submitted to IEEE Trans. Inform. Theory, 2007.

[10] M. Sikora, J. N. Laneman, M. Haenggi, D. J. Costello, and T. Fuja, "Bandwidth- and power-efficient routing in linear wireless networks," IEEE Trans. Inform. Theory, vol. 52, pp. 2624-2633, June 2006.

[11] L. Grokop and D. Tse, "Spectrum sharing between wireless networks," to appear IEEE Infocom, April 2008.

[12] P. Kyasanur, S. Jungmin, C. Chereddi, and N. Vaidya, "Multichannel mesh networks: challenges and protocols," IEEE Wireless Communications, vol. 13, no. 2, pp. 30-36, 2006. 
[13] Y. Yuan, P. Bahl, R. Chandra, T. Moscibroda, and Y. Wu, "Allocating dynamic time-spectrum blocks in cognitive radio networks," in MobiHOC, 2007.

[14] D. Stoyan, W. Kendall, and J. Mecke, Stochastic Geometry and Its Applications. John Wiley and Sons, 1996.

[15] G. Caire, G. Taricco, and E. Biglieri, "Optimum power control over fading channels," IEEE Trans. Inform. Theory, vol. 45, no. 5, pp. 1468-1489, July 1999.

[16] E. S. Sousa and J. A. Silvester, "Optimum transmission ranges in a direct-sequence spread-spectrum multihop packet radio network," IEEE Journal on Sel. Areas in Communications, vol. 8, no. 5, pp. 762-71, June 1990.

[17] S. Weber, X. Yang, J. G. Andrews, and G. de Veciana, "Transmission capacity of wireless ad hoc networks with outage constraints," IEEE Trans. on Info. Theory, vol. 51, no. 12, pp. 4091-4102, Dec. 2005.

[18] J. G. Andrews, S. Weber, and M. Haenggi, "Ad hoc networks: To spread or not to spread?" IEEE Comm. Magazine, vol. 45, no. 12, pp. 84-91, Dec. 2007.

[19] N. Jindal, S. Weber, and J. Andrews, "Fractional power control for decentralized wireless networks," submitted to IEEE Trans. Wireless Commun., Dec. 2007. 